\title{
REVIEWERS FOR VOLUME 16 (2015)
}

The Editor-in-Chief and the Editors wish to express sincere gratitude to the following people for acting as reviewers for papers rejected or published during 2015. Your critical comments and efforts to increase the quality of the papers are highly acknowledged.

Monica Violeta Achim, Babes Bolyai University, Romania

Hamimah Adnan, Universiti Teknologi MARA, Malaysia

Frank Agbola, The University of Newcastle, Australia

Elizabeth Agyeiwaah, Ghana Technology University College, Ghana

Noor Hazlina Ahmad, Universiti Sains Malaysia, Malaysia

Rizwan Raheem Ahmed, Indus University, Pakistan

Seyeon Ahn, Seoul National University, Republic of Korea

Iilknur Akiner, Akdeniz University, Turkey

Amer Ali Al-Atwi, Al-Muthanna University, Iraq

Erlantz Allur, University of the Basque Country, Spain

Borja Amor-Tapia, University of Leon, Spain

Alin Marius Andrieș, Alexandru Ioan Cuza University of Iasi, Romania

Jurgita Antuchevičiené, Vilnius Gediminas Technical University, Lithuania

Seyfettin Artan, Karadeniz Technical University, Turkey

Çağnur Balsari, Dokuz Eylül University, Turkey

Audrius Banaitis, Vilnius Gediminas Technical University, Lithuania

Eleonora Bartoloni, Italian Institute of Statistics, Italy

Agnieszka Izabela Baruk, Lodz University of Technology, Poland

Vijaya Baskaran, Kristu Jayanti College, India

Amparo Baviera-Puig, Universitat Politècnica de València, Spain

Nemanja Berber, University of Novi Sad, Serbia

Joanna Blach, University of Economics in Katowice, Poland
Elisio Brandao, University of Porto, Portugal

Peter Burger, Technical University of Kosice, Slovakia

Andrzej Buszko, University of Warmia and Mazury, Poland

Yiming Cai, South China Normal University, China

Burak Çapraz, Ege University, Turkey

José Carlos Casillas Ignacio Castro, University of Seville, Spain

Adam H. Cave, Kyungsung University, Republic of Korea

Şaban Çelik, Yaşar University, Turkey

Daniele Cerrato, Università Cattolica del Sacro Cuore, Italy

Kuei-Lun Chang, Ming Chuan University, Taiwan

Wei-Lun Chang, Tamkang University, Taiwan

Peerayuth Charoensukmongkol, Texas A\&M International University, United States

Hong Long Chen, National University of Tainan, Taiwan

Chun-Da Chen, Tennessee State University, United States

You-hua Chen, South China Agricultural University, China

Paul Moon Sub Choi, Ewha Womans University, Republic of Korea

Jaideep Chowdhurry, James Madison University, United States

Amalia Çipi, University of Vlora "Ismail Qemali", Albania

Dragan Ćoćkalo, University of Novi Sad, Serbia

Emilio Congregado, University of Huelva, Spain

Dan Dacian Cuzdriorean, Babes-Bolyai University, Romania

Alexandre Di Miceli da Silveira, University of Sao Paulo, Brazil 
Svetlana Dabic, University of Belgrade, Serbia

Marina Dabic, University of Zagreb, Croatia

Dan Cristian Dabija, Babes-Bolyai University, Romania

Silvo Dajčman, University of Maribor, Slovenia

Luciana Dalla Valle, University of Plymouth, United Kingdom

Ignacio Danvilla-del Valle, Complutense University of Madrid, Spain

Felipa de Mello-Sampayo, University Institute of Lisbon, Portugal

Blanca L. Delgado-Márquez, University of Granada, Spain

Mehmet Ö Demir, Alanya Mühendislik Fakültesi, Turkey

Abdelkader Derbali, Higher Institute of Management of Sousse, Tunisia

S. G. Deshmukh, Indian Institute of Technology Delhi, India

Eurico Brilhante Dias, University Institute of Lisbon, Portugal

Nieves L. Díaz-Díaz, University of Las Palmas de Gran Canaria, Spain

Banu Dincer, Galatasaray University, Turkey

Caner Dincer, Galatasaray University, Turkey

Hung-bin Ding, Loyola University Maryland, United States

Volkan Dogan, Eskisehir Osmangazi University, Turkey

Krzysztof Dziekonski, Bialystok University of Technology, Poland

Wolfgang Eber, Technische Universität München, Germany

Ebru Esendemir, Yasar University, Turkey

Mansour Esmaeil Zaei, Panjab University, American Samoa

J. Augusto Felício, Technical University of Lisbon, Portugal

Rui Fernandes, Aveiro University, Portugal

João J. Ferreira, University of Beira Interior, Portugal

Sibel Fettahoglu, Kocaeli University Faculty of Economics and Business Administration, Turkey

Walter Leal Filho, Hamburg University of Applied Sciences, Germany

Antonio Freitas, Federal University of Bahia, Brazil
Emilio Galdeano-Gómez, Universidad de Almería, Spain

Ernesto Galvis, National University of Colombia, Colombia

Azmat Gani, Sultan Qaboos University, Oman

Maria Teresa Garcia, Universidade de Lisboa, Portugal

Monika Golonka, Kozminski University, Poland

M. Rosario González-Rodríguez, Universidad de Sevilla, Spain

Sergej Gricar, School of Business and Management Novo mesto, Slovenia

Hima Gupta, Jaypee Business School, India

Hong-Youl Ha, Dongguk University, Korea

Heiko Haase, University of Applied Sciences Jena, Germany

Brigit Hagen, University of Pavia, Italy

Mary Hardie, University of Western Sydney, Australia

Iñaki Heras-Saizarbitoria, University of the Basque Country, Spain

Jonathan C. Ho, Yuan Ze University, Taiwan

Max Hogeforster, Baltic Sea Academy, Germany

Jens Leth Hougaard, University of Copenhagen, Denmark

Jui-Cheng Hung, Chinese Culture University, Taiwan

Supachart Iamratanakul, Kasetsart University, Thailand

Seda Yıldırım, Kocaelı University, Turkey

Hyung Rok Yim, Hanyang University, Republic of Korea

Mastura Jaafar, University Sains Malaysia, Malaysia

Charbel José Chiappetta Jabbour, University of Sao Paulo Business School, Brazil

Alireza Jalali, University Sains Malaysia, Malaysia

Karel Janda, University of Economics, Czech Republic

Gholamreza Jandaghi, University of Tehran, Iran

Mauricio Jara, Universidad de Chile, Chile

Carlos Jardon, University of Vigo, Spain

Syed Jawad Hussain, Iqra University, Pakistan 
Ana Isabel Jiménez-Zarco, Open University of Catalonia, Spain

Pornsit Jiraporn, Pennsylvania State University, United States

Cengiz Kahraman, Istanbul Technical University, Turkey

Veera Pandiyan Kaliani Sundram, University of Technology Mara, Malaysia

Sun A Kang, Chungnam National University, Republic of Korea

Ece C. Karadağll, Cankaya University, Turkey

Edvins Karnitis, Public Utilities Commission, Latvia

Salih Turan Katircioglu, Eastern Mediterranean University, Turkey

Aynur Kazaz, Akdeniz University, Turkey

Javad Khamisabadi, Young Researchers and Elite Club, Islamic Republic of Iran

Akin Kocak, Ankara University, Turkey

Žiga Kotnik, University of Ljubljana, Slovenia

Algirdas Krivka, Vilnius Gedinimas Technical University, Lithuania

Vilmante Kumpikaite, Kaunas University of Technology, Lithuania

Su-Hui Kuo, National Central University, Taiwan

Chen-Yin Kuo, Tung Fang Design Institute, Taiwan

Radosław Kurach, Wroctaw University of Economics, Poland

Chung-Jung Lee, Ming Chuang University, Taiwan

Eric Lien, Thompson Rivers University, Canada

Peide Liu, Shandong Economic University, China

Eva Livavcová, Prešov University in Prešov, Slovakia

Edmundo Lizarzaburu, Universidad ESAN, Peru

Maria Encarnacion Lucas-Perez, University of Murcia, Spain

Hannan Amoozad Mahdiraji, Islamic Azad University, Islamic Republic of Iran

David Mayes, University of Auckland, New Zealand

Jennifer Martinez-Ferrero, University of Salamanca, Spain
Pedro Martínez-Solano, University of Murcia, Spain

José Martins, University of Trás-os-Montes e Alto Douro, Portugal

Mediha Mezhoud, Faculty of Science Economics and Management of Tunis, Tunisia

Adriana Mohd Rizal, Universiti Teknologi Malaysia, Malaysia

Anna Maria Moisello, University of Pavia Italy

Marta Muñoz-Guarasa, Universidad de Jaén, Spain

R. Renuka Murugappan, SASTRA University, India

Dilip Nandkeolyar, Thapar University, India

Maria Manuela Santos Natário, Guarda Polytechnics Institute, Portugal

Zlatko Nedelko, University of Maribor, Slovenia

Collins C. Ngwakwe, University of Limpopo, South Africa

Pu-yan Nie, Jinan University, China

Lina Novickyte, Vilnius University, Lithuania

Svetoslav Novkov, JAR Cluster, Bulgaria

Osman Kürşat Onat, Mehmet Akif Ersoy University, Turkey

Yucel Ozturkoglu, Yasar university, Turkey

Eunil Park, Korea Advanced Institute of Science and Technology, Republic of Korea

Jerzy Paslawski, Poznan University of Technology, Poland

Darja Peljhan, University of Ljubljana, Slovenia

Orlando Petiz Pereira, University of Minho, Portugal

Javier Perote, University of Salamanca, Spain

Lina Pileliene, Vytautas Magnus University, Lithuania

Tatjana Polajeva, Tallinn University of Technology, Estonia

Burçak Polat, Eastern Mediterranean University, Turkey

Wojciech Przychodzen, Kozminski University, Poland

R. Seranmadevi, KSR College of Technology, India 
Marija Radosavljevic, University of Nis, Serbia

Mirjana Radovic, Akamai University, USA

Samuel Raisanen, Central Michigan University, United States

K. S. Reddy, Xi'an Jaotong University, China

Ignacio Requejo, Universidad de Salamanca, Spain

Azimi Reza, Ministry of Industry, Mine and Trade, Islamic Republic of Iran

Rosa Maria Rio, Escuela Universitaria de Ingenieria, Spain

Marko Ropret, University of Primorska, Slovenia

Nicolas Sahuguet, Hec Montréal, Canada

Abdoul G. Sam, The Ohio State University, United States

R. Dhinakaran Samuel, Loyola Institute of Technology, India

Ozan Mehmet Saray, Inonu University, Turkey

Jarmila Sebestova, Silesian University in Opava, Czech Republic

Zeliha Seçkin, Aksaray University, Turkey

Antoni Seguí Alcaraz, Valencia University, Spain

Muhammad Shahbaz COMSATS Institute of Infromation Technology, Pakistan

Azra Shamim, University Malaya, Malaysia

Dhiaa Shamki, University Utara Malaysia, Malaysia

Jian-Jun Shu, Nanyang Technological University, Singapore

Rosario Silva, IE Business School, Spain

Rasa Smaliukiene, Vilnius Gediminas Technical University, Lithuania

Vinicius Sobreiro, University of Brasilia, Brazil

Wei Song, Thompson Rivers University, Canada

Kayhan Tajeddini, Lund University, Sweden

Hidayet Tiftik, Turgut Ozal University, Turkey

Michal Tkáč, The University of Economics in Bratislava, Slovakia

Mehmet Top, Hacettepe University, Turkey

Marián Tóth, Slovak University of Agriculture, Slovakia

Francesco Trivieri, University of Calabria, Italy
Eleuterio Vallelado, University of Valladolid, Spain

$\boldsymbol{T} \boldsymbol{N}$ Varma, National Institute of Technology, India

Tiia Vissak, University of Tartu, Estonia

Abdollah Vosoughi Niri, Shahid Sattary University, Islamic Republic of Iran

Mladen Vukomanovic, University of Zagreb, Croatia

Kuo-Yan Wang, Xiamen University Tan Kah Kee College, China

X. Henry Wang, University of MissouriColumbia, American Samoa

Scott Keith Weaven, Griffith University, Australia

Joanna Wyrobek, Cracow University of Economics, Poland

Pei-Shan Wu, Ching Yun University, Taiwan

Adriana Zait, University Alexandru Ioan Cuza Iasi, Romania

Jijun Zhang, Southwest Petroleum University, China

Daiva Žostautienè, Kaunas University of Technology, Lithuania 